\title{
Sustainable Vegetable Crop Supply Problem with Perishable Stocks
}

\author{
Alysson M. Costa, ${ }^{1 *}$ Lana Mara R. dos Santos, ${ }^{2}$ Douglas J. Alem, ${ }^{3}$ Ricardo H. S. Santos ${ }^{4}$ \\ ${ }^{1}$ Instituto de Ciências Matemáticas e de Computação - Universidade de São Paulo, Brazil \\ ${ }^{2}$ Departamento de Matemática - Universidade Federal de Viçosa, Brazil \\ ${ }^{3}$ Departamento de Engenharia de Produção - Universidade Federal de São Carlos, Brazil \\ ${ }^{4}$ Departamento de Fitotecnia - Universidade Federal de Viçosa, Brazil
}

\begin{abstract}
In this paper, we deal with a vegetable crop supply problem with two main particularities: (i) the production must respect certain ecologically-based constraints and (ii) harvested crops can be stocked but only for a limited period of time, given that they are perishable. To model these characteristics, we develop a linear formulation in which each variable is associated to a crop rotation plan. This model contains a very large number of variables and is therefore solved with the aid of a column generation approach. Moreover, we also propose a two-stage stochastic programming with recourse model which takes into consideration that information on the demands might be uncertain. We provide a discussion of the results obtained via computational tests run on instances adapted from real-world data.
\end{abstract}

Keywords: linear programming, crop demand, crop rotation, column generation, two-stage stochastic programming with recourse.

\section{Introduction}

Recent years have seen a raise in sustainability awareness. Indeed, a growing number of citizens and governments are showing increasing concern with regards to this issue, which has reached a priority status in places such as the media and political campaigns. In particular, in what concerns agricultural production and distribution models, a rising number of consumers are now inclined to choose products originating from more social-friendly and ecologically-based agricultural systems, such as equitable, fair-trade, organic, biodynamical, etc. Altieri (1995); Gliessman (2000); Makatouni (2002).

These are alternative models to the conventional agricultural production and distribution system which is based mainly in large monocultures and industry-based inputs. Although the conventional paradigm claims a reduction in food production costs, there are important advantages associated with small-scale farming systems: from a ecological point of view, there is a less intense use of synthetic fertilizers, pesticides and polluting oil-based resources. From a social point of view, the significantly

\footnotetext{
${ }^{*}$ Corresponding author. Postal address: Av. do Trabalhador São-carlense, 400, CP. 668, São Carlos - SP, Brazil. CEP: 13560-970. Tel.: +55 1633738164; Fax: +55 1633739175. E-mail address: alysson@icmc.usp.br
} 
lower capital requirements in small-scale farming systems do not exclude small farmers from the process. These farmers tend to stimulate local economies by purchasing and selling locally, and creating more jobs (Ong'wen and Wright; 2007, and references therein).

These new production models give rise to different planning problems. In this context, this paper focuses on a situation faced by some small family farmers of vegetable crops in Brazil. These producers usually own small cropping areas yielding small and discontinuous productions. The size and characteristics of the production make it harder for them to individually invest in the necessary cleaning and packing house structures and to establish permanent links with the consumer market. A solution is found in the creation of associations or cooperatives, for instance. These organizations gather many small farmers, and promote the packing, standardization and distribution of the productions to the consumers, according to an expected demand. They must coordinate the production, deciding when and where to grow each crop.

In addition, in the case dealt with in this paper, the production plans must respect technicalecological constraints which allow a more sustainable production and a higher market value for the products. In this case, these constraints are basically of three types: a) crops of the same botanical family can not be grown in sequence, in the same piece of land, b) a green manure crop, usually some legume species, must be grown periodically, and c) the land must lie fallow for a specific amount of time.

We call the problem of deciding the division of land, and production and distribution plans presented above the Sustainable Vegetable Crop Supply Problem with Perishable Stocks (SVCSPPS), whose main characteristics are summarized below:

1. A known demand for each crop exists.

2. Each crop has specific earliest and latest planting times in the season that must be respected.

3. The time between planting and harvesting varies for the different crops.

4. Ecologically-based constraints forbidding the growth of crops of the same botanical family in sequence must be respected. These constraints also impose fallows and the periodical growth of green manure crops.

5. Harvested crops can be stocked for a limited and different amount of time, with associated losses.

Problems with some of characteristics 1-5 have already been proposed in the literature. Indeed, agricultural planning problems have been in the very origins of Operations Research. As early as 1939, Kantorovich developed a linear model to select the optimal partition of heterogeneous land to maximize the total production of crops (Kantorovich; 1960, translated from the Russian original, dated 1939). On a similar line, Hildreth and Reiter (1951), in the pioneering 1949 Chicago Conference on Linear Programming Applications, proposed the growing of crops simultaneously as a way to reduce the need for resources (water, labor, etc.), as crops have different needs through time. The authors used the notion of crop rotation plans, sequences of crops that should be planted one after another indefinitely. The set of possible rotation plans was defined a priori. Later, El-Nazer and McCarl (1986) developed a linear optimization model that eliminated the need of pre-determined rotations under the assumption that the yields of a crop only depend upon the crops grown in the same piece of land during the previous three years. 
Haneveld and Stegeman (2005) have proposed a model to obtain crop rotation plans based on the idea of explicitly forbidding some sequences of crops. Detlefsen and Jensen (2007) have assumed that the amount of land to be used with each crop, at each year, is known, which has enabled the modeling of the problem to be done with the aid of conservation flow constraints. More recently, Alfandari et al. (2010) propose a mixed-integer linear optimization model to obtain crop rotation plans with demand constraints and incompatibility constraints between cultivation and fallow state on a land plot. In addition, some researchers concentrate on the development of decision-aid tools to evaluate a given crop rotation plan (Jones et al.; 2003; Stöckle et al.; 2003; Bachinger and Zander; 2006, e.g.). These tools might also be useful to help implement and adapt optimization-obtained crop rotation plans in practical contexts.

Sustainability constraints of the type described in the introduction have been used by Santos, Michelon, Arenales and Santos (2010). The authors proposed a 0-1 linear optimization model for the planning of crop rotations with spatial constraints: crops of the same botanical family are not allowed to grow simultaneously in adjacent plots. Santos, Costa, Arenales and Santos (2010) have introduced the idea of a "supply problem" with the inclusion of known demands. The problem dealt with in that paper copes with characteristics 1-4 presented above, but fails to consider the presence of stocks.

Vegetable crops are usually sent to packing houses after harvesting. In the packing houses the vegetables are cleaned, packed and stored in cold chambers until delivery. Due to the high perishableness of many vegetable crops, however, they can only remain in stock for a limited amount of time and, even so, with associated losses. The possibility of post-harvest storage defines a more complex problem. In addition, considering a period of few days, the possibility of storage might provide alternative crop rotation plans that still meet the demand (eventually with stocked products) and is able to maximize revenue.

The goal of this paper is to study the effect of the introduction of post-harvest storage in the previously addressed Sustainable Vegetable Crop Supply Problem (Santos, Costa, Arenales and Santos (2010)). The post-harvest storage allows products to be stocked, while respecting maximum stocking periods and considering the associated losses. The modeling is done with the aid of "time-dependent variables" Gouveia (1999); Costa et al. (2009) which, in this context, count the number of periods a given product has been kept in stock.

Also, this paper presents an alternative model to consider the cases where the demand is uncertain. In order to take randomness into account, we model the demand using a set of discrete scenarios with given probabilities and formulate the resulting problem as a two-stage stochastic programming with recourse model. To evaluate how beneficial the use of stochastic models can be in this specific situation, two measures of uncertainty effects commonly used in stochastic programming are further analyzed. In the literature, stochastic programming has been successfully applied in diverse operations research problems. In particular, Ahumada and Villalobos (2009) pointed out the the need for stochastic models to deal with the tactical planning of perishable and non-perishable agri-foods.

The remainder of this article is divided as follows. A linear model for the SVCSPPS is proposed in the following section. This model presents a number of variables that grows exponentially with the problem size and is therefore solved via a column generation approach, which is presented in Section 3. An extension of this model that takes into consideration the fact that the demand is uncertain is developed in Section 4. Computational results on instances inspired from real-world data are discussed in Section 5. This paper ends with some conclusions and future work directions in Section 6. 


\section{Mathematical model}

In an agricultural production system, a crop rotation can be defined as the sequence of crops that should be planted, one after another, in a given area during a time interval, say $\mathrm{T}$ years. A crop rotation schedule is a planting calendar of $\mathrm{M}$ periods (in which $\mathrm{T}$ was divided) for each crop in the rotation. In this section, we extend the crop rotation schedule with known demands model proposed by Santos, Costa, Arenales and Santos (2010), to introduce the possibility of stocking harvested crops. By defining variables:

$\lambda_{k}$ size of plot associated with crop rotation plan $k$,

and parameters:

A total available arable land;

$M \quad$ number of periods in a crop rotation schedule;

$a_{i j}^{k} \quad$ amount of crop $i$ harvested in period $j$ in crop rotation plan $k$, per unity of area;

$c_{k}$ return associated with crop rotation plan $k$;

$d_{i j} \quad$ demand of crop $i$ in period $j$;

$K$ set of possible crop rotation plans.

Santos, Costa, Arenales and Santos (2010) have written the original model as:

$$
\begin{gathered}
\operatorname{Max} \sum_{k=1}^{K} c_{k} \lambda_{k} \\
\text { subject to } \quad j=1, \ldots, M, \\
\sum_{k \in K} a_{i j}^{k} \lambda_{k} \geq d_{i j}, \quad j=1, \quad \\
\sum_{k \in K} \lambda_{k} \leq A, \\
\lambda_{k} \geq 0 \quad k \in K .
\end{gathered}
$$

Constraints (2) guarantee that the demand is supplied, by stating that the production for each crop $i$, at each period $j$ must be greater than the associated demand $d_{i j}$. The production of crop $i$ at period $j$ is given by the sum, for all used crop rotation plans, of the area dedicated to the plan, $\lambda_{k}$, multiplied by the associated productivity of crop $i$ at period $j$. Constraints (3) limit the area to be used to the area actually available.

Formulation (1)-(4) does not model the possibility of keeping some of the crops in stock to serve the demand of future periods. To overcome this limitation, we define variables:

$I_{i j}^{w}$, stock of product $i$ available at period $j$ which has been produced $w$ periods previously,

$n_{i j}^{w}$, quantity of crop $i$ delivered at period $j$ using stocks $I_{i j}^{w}$.

$\theta_{i j}^{+}$, quantity of crop $i$ delivered at period $j$ that exceeds the demand $d_{i j}$.

$\theta_{i j}^{-}$, unmet demand of product $i$ in period $j$.

In both sets of variables $I_{i j}^{w}$ and $n_{i j}^{w}$, index $w$ indicates the age of the product. Index $w=0$ indicates a product that has just been harvested. The idea of using such time-indexes is adapted from existing developments in the literature in the context of hop-constrained spanning trees Gouveia (1999); Costa et al. (2009). 
Production balance:

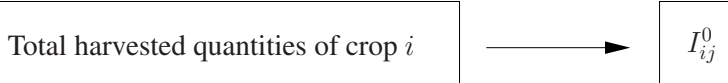

Stock balance:

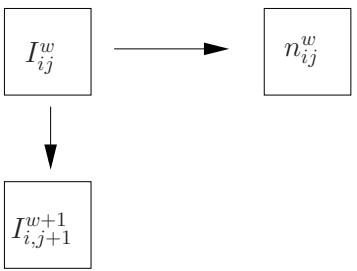

Demand supply:

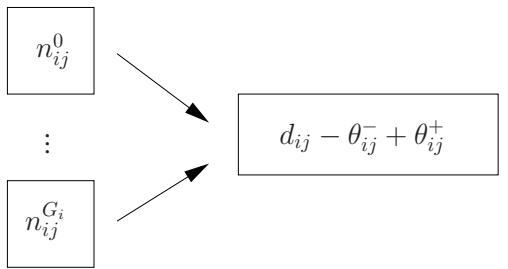

Figure 1: Relations between variables for crop $i$ at period $j$

At each period, the available stocks (of different ages) can either be used to serve the current demand or be kept in stock for one additional period. To model the fact that each product can only be stocked for a limited amount of time, it suffices to limit index $w$ to appropriate values. For example, if a crop can be stocked for 3 periods, only the associated variables with indexes $w=0, \ldots, 4$ should exist, the index 4 being associated with spoiled product. In the general case, parameter $G_{i}$ represents the number of periods a given crop $i$ can be kept in stock. Figure 1 illustrates the general idea.

The total harvested quantities of crop $i$, at period $j$, is modeled by variables $I_{i j}^{0}$ (Production balance). This quantity can either be used to meet the demand of period $j$, by means of variables $n_{i j}^{0}$ or be kept in stock, in which case it will appear (discounted the losses) as variable $I_{i, j+1}^{1}$ in the next period. The same is valid for values of $w$ other than zero (Stock balance). All quantities $n_{i j}^{w}$ are used to meet the current demand (Demand supply). Variables $\theta_{i j}^{-}$and $\theta_{i j}^{+}$model the unmet demand or the quantity sold above the demand, respectively.

With the following additional notation:

$c_{i j}$ revenue associated with product $i$, when delivered at period $j$ to meet the contracted demand;

$c_{i j}^{+} \quad$ revenue associated with the quantity of product $i$ delivered at period $j$ exceeding the demand;

$r_{i w}$ percentage loss incurring in product $i$ of age $w$ periods, if stocked for one more period;

$C \quad$ set of crops, not including those associated with green manuring;

The SVCSPPS model can thus be written as:

$$
\operatorname{Max} \sum_{i \in C} \sum_{j \in M} c_{i j}\left(d_{i j}-\theta_{i j}^{-}\right)+c_{i j}^{+} \theta_{i j}^{+}
$$

subject to

$$
\begin{gathered}
\sum_{k \in K} a_{i j}^{k} \lambda_{k}=I_{i j}^{0}, \quad i \in C, j=1, \ldots, M, \\
\sum_{k \in K} \lambda^{k} \leq A, \\
\left(1-r_{i w}\right)\left(I_{i j}^{w}-n_{i j}^{w}\right)=I_{i, j+1}^{w+1}, \quad i \in C, j=1, \ldots, M, w=0, \ldots, G_{i}, \\
\sum_{w=0}^{G_{i}} n_{i j}^{w}=d_{i j}-\theta_{i j}^{-}+\theta_{i j}^{+}, \quad i \in C, j=1, \ldots, M,
\end{gathered}
$$




$$
\begin{array}{rl}
\lambda_{k} \geq 0 & k \in K, \\
\theta_{i j}^{-}, \theta_{i j}^{+} \geq 0 & i \in C, j=1, \ldots, M, \\
n_{i j}^{w}, \geq 0 & i \in C, j=1, \ldots, M, w=0, \ldots, G_{i} . \\
I_{i j}^{w} \geq 0 & i \in C, j=1, \ldots, M, w=0, \ldots, G_{i}+1 .
\end{array}
$$

The objective function (5) maximizes the profit which can be obtained with the selling of products to meet the demand, associated with parameter $c_{i j}$, or with the selling of extra production, associated with parameter $c_{i j}^{+}$. This can be seen as a generalization of the parameterized objective function mentioned in (1) which defined a fixed return, $c_{k}$, associated with a rotation plan. In the case of objective function (5), a single parameter such as $c_{k}$ can not be used to define the return of a crop rotation plan, once this return depends on the destination of the harvested crops (if they are used to supply the demand, sold as extra production or maintained in stock).

Constraints (6) associate variables $I_{i j}^{0}$ with the harvested quantities of each crop $i$ at each period $j$. Constraint (7) ensures that only the available land is used. Constraints (8) are the stock balance constraints and include a loss-factor for every additional period a product is kept in stock. These constraints can be read as follows: the quantity of stocked product $i$ at period $j$ having $w$ periods of age $\left(I_{i j}^{w}\right)$ can be either used to meet the demand of period $j$ (becoming $n_{i j}^{w}$ ) or remain in stock, appearing as $I_{i, j+1}^{w+1}$ in the following period. Note that for $w=G_{i}$, the constraints use a variable $I_{i j}^{G_{i}+1}$, which appears nowhere else in the formulation and, therefore, indicates losses (all remaining product $i$ after $G_{i}$ weeks is considered spoiled). Constraints (9) control the demand supply: the demand of product $i$ at period $j, d_{i j}$, can be supplied from fresh harvested products $\left(n_{i j}^{0}\right)$ or from stocked products with $w \leq G_{i}$ periods of age, $\left(n_{i j}^{w}, w \geq 1\right)$. Finally, Constraints (10)-(13) define the used variables.

\section{Column generation algorithm}

As any linear programming formulation, model (5)-(12) can be seen as a set of columns, which one associated with a variable. Each element of the column is the coefficient of the associated variable in the respective constraint. Model (5)-(12) has the following set of variables: $n_{i j}^{w}, I_{i j}^{w}, \theta_{i j}^{-}, \theta_{i j}^{+}$and $\lambda_{k}$. In particular, each one of variables $\lambda_{k}$ is associated with a column that contains all the information with respect to a given crop rotation plan. Indeed, a crop rotation plan $k$ generates a harvesting schedule that can be represented by parameters $a_{i j}^{k}$, the quantities to be harvested for each crop $i$, at period $j$, for each unit of area dedicated to the crop rotation plan $k$. In possession of parameters $a_{i j}^{k}$, the column associated with variable $\lambda_{k}$ can be easily written as:

$$
\text { column } \tilde{a}_{k}=\left[\begin{array}{c}
\left(a_{i j}^{k}\right) \\
1 \\
\mathbf{0}
\end{array}\right] \begin{aligned}
& \rightarrow \text { associated with constraints } \\
& \rightarrow \text { associated with constraint }
\end{aligned}
$$

In the case of the crop rotation plans defined by conditions 2-4 (see Introduction), the number of possible plans grows exponentially with the problem size. To cope with this problem, model (5)-(12) can be seen as a master program within a column generation framework Lübbecke and Desrosiers (2005). The main idea would be to solve the problem for an initial set of columns (possibly columns associated with variables $n_{i j}^{w}, I_{i j}^{w}, \theta_{i j}^{-}, \theta_{i j}^{+}$) and then, iteratively include promising columns $\tilde{a}_{k}$ until 
optimality is found.

To generate promising columns $\tilde{a}_{k}$, one needs an auxiliary problem composed by a set of constraints describing the feasible space of crop rotation plans $K$, and an objective function associated with the reduced cost of a column $\tilde{a}_{k}$ in model (5)-(12). These two developments are done in what follows. Define binary variables:

$x_{i j}$, equal to 1 if crop $i$ is planted in period $j$, and equal to 0 , otherwise,

and parameters:
NF number of botanical families;
$F(p) \quad$ set of crops belonging to botanical family $p, p=1, \ldots, N F$;
$t_{i} \quad$ production time of crop $i$, including the estimated soil preparation and harvesting times;
$I_{i} \quad$ set of planting periods for crop $i$;
$N \quad$ set of all crops;
$G \quad$ set of crops that can be used as green manure;
$n=|N|+1$ artificial crop associated with fallow. The cultivation period of this crop is the fallow length.

The technological constraints 2-4 can then be written as in Santos, Michelon, Arenales and Santos (2010):

$$
\begin{aligned}
\sum_{i=1}^{n} \sum_{r=0}^{t_{i}-1} x_{i, j-r} & \leq 1, \quad j=1 \ldots M \\
\sum_{i \in F(p)} \sum_{r=0}^{t_{i}} x_{i, j-r} & \leq 1, \quad p=1 \ldots N F, j=1, \ldots, M, \\
\sum_{i \in G} \sum_{j \in I_{i}} x_{i j} & =1, \\
\sum_{j=1}^{M} x_{n j} & =1, \\
x_{i j} \in\{0,1\}, i=1, \ldots, n, j \in I_{i}, &
\end{aligned}
$$

where, in constraints (14) and (15), non-positive indexes $j-r$ are replaced by $j-r+M$. Constraints (14) guarantee that no more than one crop is planted at a time. Constraints (15) ensure that two crops of the same botanical family can not be planted in sequence. Finally, constraints (16) and (17) force the planting of a crop associated with green manure and the presence of a time of fallow at each rotation, respectively.

Moreover, by associating dual variables $\pi_{i j}$ and $\alpha$ to (6) and (7), respectively, one can calculate the reduced cost of a column $\tilde{a}_{k}$, which is given by:

$$
\tilde{c}_{k}=\sum_{i \in C} \sum_{j \in I_{i}}-\pi_{i j} a_{i j}^{k}-\alpha
$$

Let $\left(\hat{x}_{i j}^{k}\right)$ be the $k^{\text {th }}$ feasible crop rotation schedule, i.e., satisfying (14)-(18), the associated coefficients $a_{i j}^{k}$ can be easily determined with the aid of the following parameters: 
$o_{i} \quad$ number of periods between planting and the first harvesting of crop $i$;

$p_{i r} r^{\text {th }}$ harvesting of crop $i, r=1, \ldots, t_{i}-o_{i}-1$.

These parameters define the programmed harvestings of a given crop. For example, if for crop 1, $t_{1}=4, o_{1}=1$ and $p_{11}=2 \mathrm{~kg}, p_{12}=3 \mathrm{~kg}$, then crop 1 , if planted in period 1 will yield harvest of 2 and $3 \mathrm{~kg}$ per $m^{2}$ of planted area in periods 3 and 4 , respectively.

Thus, for a given crop rotation schedule, $\left(\hat{x}_{i j}^{k}\right)$, coefficients $a_{i j}^{k}$ can be written as:

$$
a_{i j}^{k}=\sum_{r=1}^{t_{i}-o_{i}-1} p_{i r} \hat{x}_{i, j-o_{i}-r}^{k}, \quad i \in C, j=1, \ldots, M, k=1, \ldots, K .
$$

where index $j-o_{i}-r$ is replaced by $M+j-o_{i}-r$ whenever it less or equal to zero.

The goal of the auxiliary problem is to find a promising column for the master problem. The column with the largest reduced cost is, therefore, found by solving the following problem:

$$
\begin{aligned}
\operatorname{Max} & \sum_{i \in C} \sum_{j \in I_{i}}\left(\sum_{r=1}^{t_{i}-o_{i}-1}-\pi_{i, j+r+o_{i}} p_{i r}\right) x_{i j}-\alpha \\
& \text { subject to (14)-(18) }
\end{aligned}
$$

Algorithm 1 presents a pseudo-code for the implemented column generation.

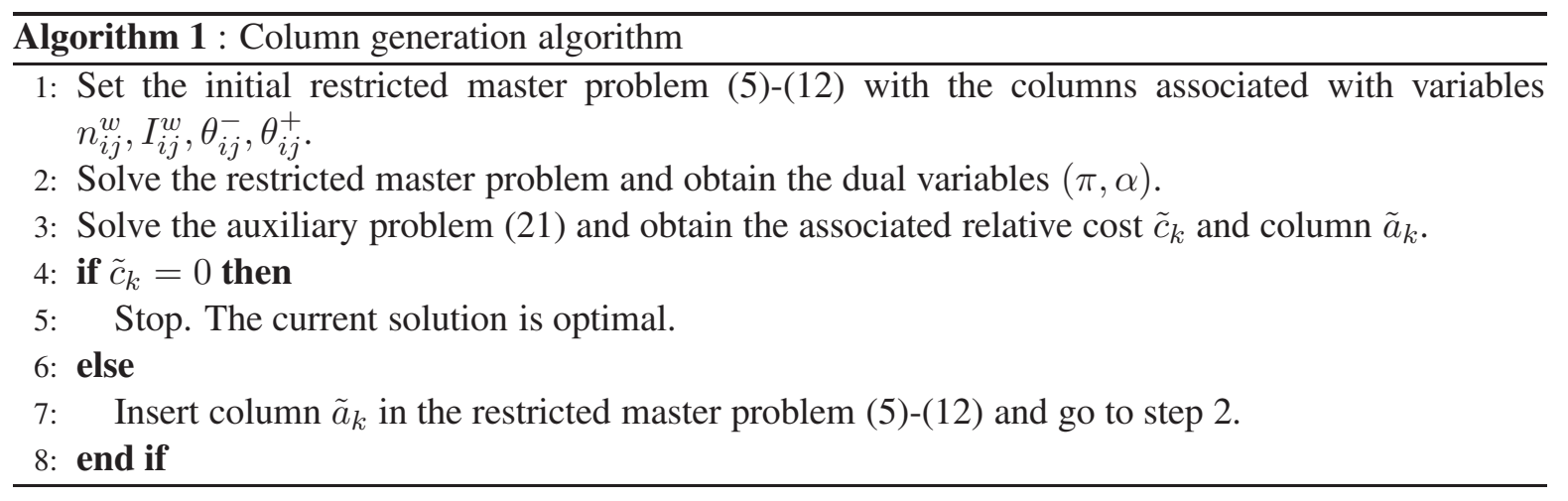

The convergence of simplex-based column generation algorithms has been the subject of much research Du Merle et al. (1999); Lübbecke and Desrosiers (2005). In this kind of algorithmic approach, it is very common that a near-optimal solution is obtained considerably fast but very little progress is obtained at each iteration, near the optimum. This is the so-called "tailing-off effect". Other frequently observed effect is that the master problem becomes harder to solve due to massive degeneracy and the fact that the dual variables end up not yielding efficient columns Du Merle et al. (1999). In Section 5, we present computational results which show that, for the proposed model, real-life problems could be solved in quite reasonable computational times and no convergence difficulties were experienced. In that section, we also analyze the effect of allowing harvested products to be stocked. Before that, in the following section, we extend model (5)-(12) to try to cope with the fact that the demands might be uncertain 


\section{A two-stage stochastic programming with recourse}

In this section we present a discussion on how model (5)-(12) can be extended to cope with uncertainties in demand. The choice of demand as the source of uncertainty seems natural in the case dealt with in this article. Indeed, although the market for ecologically-based crops is growing, it is still one of the first to be affected in situations of economical crisis or recession and, therefore, a natural uncertainty in demands do exist.

We propose a two-stage stochastic programming with recourse model to consider this situation. Stochastic programming models with recourse are adequate when decisions might be taken in two stages: part of the decision variables must be determined beforehand and part can be chosen only after a set of random variables is observed. The second set of decision variables can be used to implement corrective actions with respect to the decisions taken in the first stage. For details, the reader is referred to Dantzig (1955); Kall and Wallace (1994); Birge and Louveaux (1997).

In this paper, the stochastic demand is represented through a set of discrete outcomes $s \in \Omega=$ $\{1,2, \cdots, S\}$, called scenarios, with associated probabilities $\mathcal{P}=\left\{p_{1}, p_{2}, \cdots, p_{S}\right\}$, such that $p_{s}>0$ for all $s$ and $\sum_{s \in \Omega} p_{s}=1$. Each scenario $s$ corresponds to a particular realization of the demand and the probability of occurrence represents the likelihood of that scenario $s$, as seen by the specialists or decision makers. Consider the following aditional notation:

$d_{i j}^{s}=$ demand of crop $i$ in period $j$ under scenario $s$;

$I_{i j}^{w, s}=$ stock of product $i$ available at period $j$ which has been produced $w$ periods previously in scenario $s$;

$n_{i j}^{w, s}=$ amount of demand of product $i$ at period $j$ that is supplied using stocks $I_{i j}^{w, s}$ in scenario $s$;

$\theta_{i j}^{+, s}=$ quantity of crop $i$ sold at period $j$ that exceeds the demand $d_{i j}^{s}$ in scenario $s$;

$\theta_{i j}^{-, s}=$ non supplied demand of $i$ at period $j$ in scenario $s$.

Our aim is to find the optimal first-stage decisions regarding land allocation and the optimal secondstage decisions associated to inventory and demand fulfillment policy. The two-stage stochastic programming with recourse (or simply recourse model) can be written as follows.

$$
\operatorname{Max} \sum_{s \in \Omega} \sum_{i \in C} \sum_{j \in M} p_{s}\left[c_{i j}\left(d_{i j}^{s}-\theta_{i j}^{-, s}\right)+c_{i j}^{+} \theta_{i j}^{+, s}\right]
$$

subject to

$$
\sum_{k \in K} a_{i j}^{k} \lambda_{k}=I_{i j}^{0, s}, \quad i \in C, j=1, \ldots, M, s \in \Omega,
$$

$$
\sum_{k \in K} \lambda^{k} \leq A
$$

$\left(1-r_{i w}\right)\left(I_{i j}^{w, s}-n_{i j}^{w, s}\right)=I_{i, j+1}^{w+1, s}, \quad i \in C, j=1, \ldots, M, w=0, \ldots, G_{i}, s \in \Omega$,

$$
\begin{array}{rlrl}
\sum_{w=0}^{G_{i}} n_{i j}^{w, s}=d_{i j}^{s}-\theta_{i j}^{-, s}+\theta_{i j}^{+, s}, & & i \in C, j=1, \ldots, M, s \in \Omega, \\
\lambda_{k} \geq 0 & & k \in K, \\
\theta_{i j}^{-, s}, \theta_{i j}^{+, s} \geq 0 & i \in C, j=1, \ldots, M, s \in \Omega, \\
n_{i j}^{w, s} \geq 0 & i \in C, j=1, \ldots, M, w=0, \ldots, G_{i}, s \in \Omega, \\
I_{i j}^{w, s} \geq 0 & i \in C, j=1, \ldots, M, w=0, \ldots, G_{i}+1, s \in \Omega .
\end{array}
$$


The revised objective function (22) maximize the expected profit which can be obtained with the selling of products to meet the demand or the selling of extra production, considering all scenarios. Constraints (23) associate variables $I_{i j}^{0, s}$ with the harvested quantities of each crop $i$ at each period $j$. Since these quantities are scenario-independent, we can replace $I_{i j}^{0, s}$ by $I_{i j}^{0}$ and the set of constraints become independent of $s$. Constraints (25) are the stock balance constraints for each scenario $s$. Constraints (26) control the demand supply, also for each scenario. Constraints (27)-(29) define the domain of the second-stage decision variables.

Model (22)-(29) can be solved with a similar column generation approach described in Section 3. Initial set of columns can be now associated with variables $n_{i j}^{w, s}, I_{i j}^{w, s}, \theta_{i j}^{-, s}, \theta_{i j}^{+, s}$ and the column with the largest reduced cost is found by solving the following problem:

$$
\begin{aligned}
\operatorname{Max} & \sum_{i \in C} \sum_{j \in I_{i}} \sum_{r=1}^{t_{i}-o_{i}-1} \sum_{s \in S}-\pi_{i, j+r+o_{i}}^{s} p_{i r} x_{i j}-\alpha \\
& \text { subject to (14)-(18) }
\end{aligned}
$$

In the following section, we test the efficiency of model (22)-(29), in terms of computational burden and with the aid of measures such as the expected value of perfect information (EVPI) and the value of stochastic solution (VSS), which can give an idea of how beneficial the use of stochastic models can be in this specific situation (Birge and Louveaux; 1997; Kall and Wallace; 1994; Escudero et al.; 2007; Alem et al.; 2010, e.g.).

\section{Computational results}

In Section 2 we have proposed a linear model with a large number of variables for the SVCSPPS while in section 3 a column generation approach has been developed for its resolution. Later, in Section 4, the SVCSPPS model has been extended to cope with uncertainty in demands, with the aid of a two-stage stochastic programming with recourse. This new model has also been solved with the aid of an adaptation of the column generation developed before.

In this section, we run a series of computational tests to analyze the performance of the algorithms and the impact of allowing the maintenance of stocks and the consideration of uncertainty in demands. In order to simulate the situations where stocks are not allowed, the same models were used and the number of periods each product could be kept in stock, $G_{i}$, was set to zero for all crops.

We use a planning period of two years, divided in weeks. For each rotation plan, a fallow of 4 weeks must be respected. The instances are divided in classes according to the parameters related to the size of arable area $\left(500 \mathrm{~m}^{2}, 1000 \mathrm{~m}^{2}, 2000 \mathrm{~m}^{2}, 4000 \mathrm{~m}^{2}\right)$ and the number of crops $(10,15$ or 20$)$. In total, we have 12 groups of instances with 10 instances each. The demands for the crops were randomly generated taking into account the appropriated planting and harvesting periods.

The crop-related data is displayed in Appendix and has been obtained at an ecologically-based production unit situated in the city of Barbacena ( $21^{\circ} 13^{\prime} 30^{\prime \prime} \mathrm{S}$ and $43^{\circ} 46^{\prime} 25^{\prime \prime} \mathrm{W}, 1165$ meters above sea level ), in the countryside of Brazil. The demand for the crops in all the periods were randomly generated taking into account planting and harvesting periods of each crop and making random changes to data collected from that production unit.

The column-generation algorithm has been implemented in $\mathrm{C}++$ and both the master and the sub- 
problem have been solved with the aid of the commercial package CPLEX 10.1 ILOG (2006). A time limit of $1 \mathrm{~h}$ has been established for each instance. The tests have been carried out at a machine under Windows, with $1.66 \mathrm{GHz}$ and $2 \mathrm{~Gb}$ of RAM. For all the experiments, we fixed $c_{i j}=1$ and $c_{i j}^{+}=0$, for all $i$ and $j$. In this way, the main goal of the algorithm was to serve the contracted demand.

The results are presented in what follows and are compiled in two subsections, one for the deterministic case and the other for the stochastic case.

\subsection{Results for the deterministic model}

In order to analyze the effect of stocks, all instances have also been solved for the situation where stocks are not allowed. Tables 1 and 2 present the average results for each one of the 12 groups of instances. The tables refer to the situations when no stock is allowed and when stocking (under the conditions described in Table 7 in Appendix) is allowed, respectively. In both tables, the results are presented in terms of the time used by the algorithm, the number of plots in the final solution, the percentage of unmet demand and the extra production (how much of a product has been produced over the demand, in percentage terms). These results are presented in the columns time, \#plots, \%resid, and $\%$ extra, respectively.

\begin{tabular}{|c|c|c|rccc|}
\hline Group & A & \#crops & time(s) & \#plots & \%resid & \%extra \\
\hline 1 & \multirow{3}{*}{500} & 10 & 53.40 & 62.40 & 18.20 & 69.50 \\
2 & 15 & 96.30 & 93.80 & 43.10 & 94.90 \\
3 & & 20 & 404.10 & 112.50 & 66.60 & 96.40 \\
\hline 4 & & 10 & 15.30 & 69.80 & 3.60 & 56.30 \\
5 & \multirow{2}{*}{1000} & 15 & 184.40 & 200.70 & 16.10 & 89.80 \\
6 & & 20 & 181.90 & 121.0 & 46.60 & 99.20 \\
\hline 7 & \multirow{3}{*}{2000} & 10 & 2.70 & 40.00 & 0.10 & 39.10 \\
8 & & 20 & 39.40 & 189.20 & 0.90 & 72.20 \\
9 & & 20 & 349.10 & 3.40 & 91.10 \\
\hline 10 & & 10 & 1.10 & 25.60 & 0.00 & 25.60 \\
11 & 4000 & 15 & 19.90 & 109.50 & 0.00 & 55.10 \\
12 & & 20 & 79.70 & 218.40 & 0.00 & 68.70 \\
\hline
\end{tabular}

Table 1: Results when no stock is allowed

\begin{tabular}{|c|c|c|rccc|}
\hline Group & A & \#crops & time(s) & \#plots & \%resid & \%extra \\
\hline 1 & \multirow{3}{*}{500} & 10 & 52.60 & 52.60 & 17.90 & 77.50 \\
2 & 15 & 99.70 & 90.40 & 43.00 & 97.30 \\
3 & & 20 & 406.80 & 110.40 & 66.60 & 97.60 \\
\hline 4 & & 10 & 11.60 & 38.70 & 3.50 & 67.30 \\
5 & \multirow{2}{*}{1000} & 15 & 272.40 & 184.70 & 15.90 & 93.20 \\
6 & & 20 & 220.70 & 120.20 & 46.50 & 99.90 \\
\hline 7 & \multirow{2}{*}{2000} & 10 & 2.50 & 21.40 & 0.10 & 49.40 \\
8 & 15 & 154.40 & 155.40 & 0.60 & 75.40 \\
9 & & 20 & 918.30 & 313.40 & 2.80 & 93.50 \\
\hline 10 & \multirow{4}{*}{4000} & 10 & 1.10 & 15.80 & 0.00 & 40.80 \\
11 & & 20 & 61.10 & 105.40 & 0.00 & 72.80 \\
12 & & & & & & \\
\hline
\end{tabular}

Table 2: Results when stock is allowed according to the conditions presented in Table 7

The computational experiments indicate that the proposed algorithm was able to find optimal solutions very quickly, both for the situations with and without stocks. In general terms, the convergence became harder to achieve as the number of crops increased but seemed independent on the planting 
areas considered. Some preliminary tests were run by varying some other parameters (such as $G_{i}$ or the crop productivities) and the computational times remained stable.

By comparing the results in Tables 1 and 2, it is possible to see that enabling stocks was generally responsible for reducing the unmet demand while increasing extra production. The results confirm our hypothesis that the possibility of keeping some products in inventory would introduce a higher level of flexibility, allowing the obtention of better solutions. It is also noteworthy that the computational burden of the new model was not significantly higher. This shows that the implemented column generation algorithm is flexible and sufficiently efficient to deal with this more complex model.

\begin{tabular}{|c|cccc|cccc|ccc|}
\hline & \multicolumn{4}{|c|}{ Without stock } & \multicolumn{6}{c|}{ With stock } \\
\hline A & time(s) & \#plots & \%resid & \%extra & time(s) & \#plots & \%resid & \%extra & \%cp. plots & \%cp. resid & \%cp. extra \\
\hline 500 & 184.60 & 89.57 & 42.63 & 86.92 & 186.37 & 84.47 & 42.48 & 90.80 & -5.69 & -0.34 & 4.46 \\
1000 & 127.20 & 130.50 & 22.07 & 81.78 & 168.22 & 114.54 & 21.96 & 86.79 & -12.23 & -0.51 & 6.12 \\
2000 & 163.27 & 192.77 & 1.46 & 67.49 & 358.40 & 163.40 & 1.16 & 72.78 & -15.23 & -20.54 & 7.84 \\
4000 & 33.57 & 117.83 & 0.00 & 49.80 & 23.73 & 57.00 & 0.00 & 58.51 & -51.63 & 0.00 & 17.48 \\
\hline Average & 127.16 & 132.67 & 16.54 & 71.50 & 184.18 & 104.85 & 16.40 & 77.22 & -21.20 & -5.35 & 8.97 \\
\hline
\end{tabular}

Table 3: Summary of results

Table 3 presents a summary of the results. The average figures are presented for each value of the available arable land. We compare the results obtained with and without the possibility of stocking. The results for the case of stocks are presented in relative terms with respect to the case without stocks. This can be seen in columns \%cp. plots, \%cp. resid and \%cp. extra which indicate the percentage comparative number of plots, unmet demand and extra production. From the table, it becomes clear the enabling stocks was responsible for reducing the unmet demand while still obtaining an increase in the extra production. In average terms, a reduction of $5.35 \%$ was obtained in the unmet demand with an increase of $8.97 \%$ in the extra production. Note, however, that the value $5.35 \%$ is mainly due to the large reduction obtained for the small residual demand of the case with $A=2000$. Considering the absolute average values without and with the use of stocks (16.54 and 16.40, respectively), a relative average reduction of $0.85 \%$ is observed. If the same calculations are done for the absolute average values corresponding to extra production, the percentage increase is still of about $8 \%$.

Table 3 also shows that the solutions obtained when stocks were allowed had a smaller number of plots. This characteristic is particularly desired since solutions with a very high number of plots might be impractical in real-life situations Santos, Costa, Arenales and Santos (2010).

\subsection{Results for the stochastic model}

To analyze the effect of considering the uncertainty in demands via the recourse model presented in (22)-(29), we run a series of additional computational tests. Three other scenarios were generated, considering increases in demand of $20 \%, 50 \%$ and $80 \%$ from the data of demand used in the tests for the deterministic problem. Again, two situations were considered: with and without the possibility of keeping stocks.

When stochastic characteristics are incorporated in a model, it may be important to determine if the recourse model is really justified or, on the contrary, if the uncertainty can be ignored. To properly investigate this, two measure of uncertainty effects, namely, the expected value of perfect information (EVPI) and the value of stochastic solution (VSS) are reported in this paper. Both measures are compared to the optimal objective function value of the stochastic model, namely, $R P$ value. 
The measure EVPI indicates the maximum amount the decision maker is willing to pay in order to obtain the value of a random variable before making his decision Birge and Louveaux (1997); Avriel and Williams (1970). It is computed by evaluating the recourse model and the 'wait-and-see' solutions, as follows: (1) solve one problem for each scenario $s$ and obtain the so called wait-and-see solutions, where the first stage decisions are taken to optimize a single scenario; (2) calculate the expectation over the set of scenarios of the wait-and-see solutions, WS; (3) compute the estimated value of perfect information as $E V P I=W S-R P$.

The measure VSS represents the added value of using a stochastic model Birge (1995). It compares the solution of the recourse model with the deterministic solution obtained by replacing all random variables by their expectations. VSS can be computed as follows: (1) solve the mean scenario problem, $E V$, where the random variables are replaced by their expectations; (2) solve the problem with the first stage variables fixed according to $E V$ optimal solution, and obtain the optimal value $E E V$; (3) determine $V S S=R P-E E V$. If $V S S \leq \epsilon$ (where $\epsilon$ is a given tolerance), then the stochastic model can be approximated by the $E V$ problem. Otherwise, there is a profit in using the stochastic model instead of considering the expected value problem. It is also possible to determine $E E V$ from the solution of the most probable scenario Liua et al. (2009) or the scenario that generates the minimum total revenue in a worst case perspective, for instance.

For both measures EVPI and VSS, we can also define relative statistics, as follows: $V S S r=(R P-$ $E E V) / R P$ and $E V P I r=(W S-R P) / R P$.

Table 4 and 5 summarizes the obtained results for the cases without and with stocks, respectively. In both tables, we present, for each group of instances, the results in terms of the time spent by the algorithm for solving the recurse problem (time), the percentage of unmet demand (\%resid), the percentage of production over the demand (\%extra), EVPI(EVPIr) and VSS(VSSr) in percentage.

\begin{tabular}{|c|c|c|rccc|cccc|}
\hline Group & $A$ & crops & time (s) & \#plots & \%resid & \%extra & EVPI & EVPIr & $V S S$ & $V S S r$ \\
\hline 1 & \multirow{3}{*}{500} & 10 & 66.80 & 71.90 & 29.40 & 78.58 & 78.88 & 0.37 & 1135.10 & 3.00 \\
2 & 15 & 108.60 & 99.80 & 56.13 & 95.98 & 121.75 & 0.20 & 1894.10 & 2.75 \\
3 & & 20 & 471.00 & 114.00 & 73.96 & 95.86 & 205.34 & 0.42 & 1457.20 & 2.23 \\
\hline Average & & & 215.47 & 95.23 & 53.16 & 90.14 & 135.32 & 0.33 & 1495.50 & 2.66 \\
\hline 1 & \multirow{2}{*}{1000} & 10 & 41.30 & 79.10 & 9.80 & 66.66 & 780.77 & 0.78 & 1691.80 & 2.79 \\
2 & 15 & 166.00 & 155.80 & 29.15 & 90.70 & 1413.10 & 1.16 & 2949.10 & 2.48 \\
3 & & 20 & 215.22 & 127.33 & 60.95 & 99.06 & 182.32 & 0.12 & 4075.20 & 2.78 \\
\hline Average & & & 140.84 & 120.74 & 33.30 & 85.47 & 792.05 & 0.69 & 2905.40 & 2.68 \\
\hline 1 & \multirow{2}{*}{2000} & 10 & 24.40 & 70.70 & 1.39 & 53.07 & 178.20 & 0.17 & 4091.80 & 4.08 \\
2 & 15 & 600.00 & 294.00 & 7.7505 & 82.162 & 564.76 & 0.29 & 9999.20 & 5.94 \\
3 & & 20 & 1318.80 & 363.10 & 23.27 & 93.54 & 806.77 & 0.35 & 10688.00 & 4.10 \\
\hline Average & & & 647.73 & 242.60 & 10.80 & 76.26 & 516.58 & 0.27 & 8259.80 & 4.71 \\
\hline 1 & \multirow{2}{*}{4} & 10 & 2.10 & 33.00 & 0.02 & 40.27 & 0.00 & 0.00 & 2348.20 & 2.69 \\
2 & 4000 & 15 & 175.10 & 195.50 & 0.15 & 64.82 & 242.73 & 0.07 & 14090.00 & 5.64 \\
3 & & 20 & 1102.70 & 436.20 & 0.67 & 76.67 & 1541.80 & 0.38 & 27770.00 & 7.45 \\
\hline Average & & & 426.63 & 221.57 & 0.28 & 60.59 & 594.84 & 0.15 & 14736.00 & 5.26 \\
\hline
\end{tabular}

Table 4: Summary of results for model (22)-(29) when stocks are not allowed

Note that, as in the deterministic case, the model with the possibility of keeping inventories usually presents smaller percentages of unmet demand. This is particularly true for the case with the larger area $A=4000$, where the percentage of unmet demand drops from $28 \%$ to $20 \%$ when stocks are allowed.

With respect to the stochastic efficiency measures EVPI and VSS, the results suggest that the resolution of a stochastic programming model can effectively help coping with the uncertainty in demands. 


\begin{tabular}{|c|c|c|c|c|c|c|c|c|c|c|}
\hline Group & $A$ & crops & time $(\mathrm{s})$ & \#plots & \%resid & \%extra & $E V P I$ & EVPIr & $V S S$ & $V S S r$ \\
\hline 1 & \multirow{3}{*}{500} & 10 & 71.00 & 64.30 & 28.79 & 84.24 & 76.96 & 0.29 & 342.28 & 2.18 \\
\hline 2 & & 15 & 145.30 & 90.60 & 55.91 & 96.72 & 153.52 & 0.24 & 391.21 & 0.80 \\
\hline 3 & & 20 & 517.70 & 112.30 & 73.89 & 96.53 & 260.60 & 0.49 & 342.69 & 0.74 \\
\hline Average & & & 244.67 & 89.07 & 52.87 & 92.49 & 163.69 & 0.34 & 358.73 & 1.24 \\
\hline 1 & \multirow{3}{*}{1000} & 10 & 110.10 & 64.90 & 9.04 & 66.51 & 532.69 & 0.55 & 1705.50 & 2.80 \\
\hline 2 & & 15 & 222.00 & 145.90 & 28.52 & 90.04 & 1270.59 & 1.02 & 1578.80 & 1.60 \\
\hline 3 & & 20 & 288.22 & 122.44 & 60.88 & 99.44 & 243.72 & 0.17 & 766.67 & 0.52 \\
\hline Average & & & 206.77 & 111.08 & 32.81 & 85.33 & 682.33 & 0.58 & 1350.30 & 1.64 \\
\hline 1 & \multirow{3}{*}{2000} & 10 & 31.10 & 43.50 & 1.26 & 54.55 & 144.67 & 0.14 & 4374.60 & 3.89 \\
\hline \multirow[t]{2}{*}{2} & & 15 & 1511.00 & 277.10 & 7.50 & 83.04 & 427.71 & 0.21 & 8692.40 & 5.50 \\
\hline & & 20 & 1724.50 & 342.50 & 23.02 & 93.48 & 814.99 & 0.34 & 4642.80 & 2.06 \\
\hline Average & & & 1088.90 & 221.03 & 10.59 & 77.02 & 462.46 & 0.23 & 5903.30 & 3.82 \\
\hline 1 & \multirow{3}{*}{4000} & 10 & 4.30 & 21.30 & 0.01 & 39.27 & 0.00 & 0.00 & 1391.60 & 1.49 \\
\hline 2 & & 15 & 700.70 & 161.10 & 0.11 & 64.28 & 154.66 & 0.04 & 14224 & 5.89 \\
\hline 3 & & 20 & 3767.00 & 376.10 & 0.49 & 77.51 & 1126.35 & 0.28 & 27941 & 7.47 \\
\hline Average & & & 1490.70 & 186.17 & 0.20 & 60.36 & 427.00 & 0.11 & 14519 & 4.95 \\
\hline
\end{tabular}

Table 5: Summary of results for model (22)-(29) when stocks are allowed

Indeed, in most cases the positive figures for both VSS and EVPI seems to justify the use of this stochastic model instead of the simpler strategy of solving the problem for the expected demand. Exceptions are found, for example, in group 1 of area $A=4000$, for which the EVPI was null. In these cases, wait-and-see solutions are a good approximation for the recourse problem.

In all cases, both with and without the possibility of keeping products in inventory, the VSS figures were positive, with $V S S r$ reaching a gain of 5.2\% when $A=4000$ and stocks are not allowed. Moreover, VSS and VSSr values were usually higher for the cases without stocks, indicating that the ability of keeping stocks can also be used to cope with uncertainties, as it is natural to expect. Also, the average performance of VSS and VSSr was better for larger areas, suggesting that the expected problem $E V$ gives a bad approximation of the recourse problem especially in theses situations.

Finally, the figures in the table show once again the efficiency of the proposed column generation algorithm, which was still able to obtain results in computational times that can be considered reasonable for a strategic-level planning problem such as the one dealt with in this article.

\section{Conclusions}

In this paper, we have studied a vegetable crop rotation problem with demand constraints. In this problem, given a set of heterogeneous arable lands, one must decide what and when to produce, while respecting some ecologically-based production constraints. The main new feature introduced by our model is the possibility of stocking harvested crops. We use time-dependent variables to cope with the fact that products are perishable and present losses after each period in inventory. To solve the model, a column generation algorithm has been developed and tested on a set of instances adapted from real-world data. We also analyzed the effect of considering uncertainty in demands via a two-stage stochastic programming with recourse model. The results suggest that the possibility of keeping stocks increases the ability of the model to meet the demand and that it might be advantageous to take into consideration uncertainty in demands. Extensions of this research might contemplate both practical and theoretical aspects. In a practical point of view, the development of graphical interfaces and opensource versions of the developed codes is necessary in order to give more autonomy to the cooperatives 
in using the developed tools. In a more theoretical research line, one might study the consideration of risk into the model to determine robust production plans, via the use of robust optimization in the sense of Mulvey et al. (1995) or Bertsimas and Sim (2004), as well as the explicit analysis of other sources of uncertainty such as prices and, mainly, crop yields given by the stochastic nature of the weather.

\section{Acknowledgements}

The authors thank the Fundação de Amparo à Pesquisa do Estado de São Paulo (FAPESP) and the Conselho Nacional de Desenvolvimento Científico e Tecnológico (CNPq). The authors also thank the participants and organizers of the 2009 Euro Summer Institute for the discussions and comments which helped improve the quality of this article as well as the anonymous reviewers for their insightful comments and suggestions.

\section{Appendix}

For the computational experiments, we selected 23 crops which are cultivated at an organic farming unit in Barbacena, Brazil. Crops $20-23$ are used for green manuring. The discretization time is one week. Data listed in Tables 6 and 7 are approximations of the real values. The production time for each crop includes the estimated planting and harvesting periods.

\begin{tabular}{|c|c|c|c|c|c|c|}
\hline \multirow{2}{*}{\multicolumn{2}{|c|}{ Crop }} & \multirow{2}{*}{\multicolumn{2}{|c|}{ Botanic family }} & \multicolumn{2}{|c|}{ Planting period } & \multirow{2}{*}{$\begin{array}{c}\text { Production } \\
\text { time }\end{array}$} \\
\hline & & & & beginning & end & \\
\hline 1 & Crisp head lettuce & $\overline{1}$ & Asteraceae & \multicolumn{2}{|c|}{ all year round } & 7 \\
\hline 2 & Loose leaf lettuce & 1 & Asteraceae & \multicolumn{2}{|c|}{ all year round } & 7 \\
\hline 3 & Butter head lettuce & 1 & Asteraceae & \multicolumn{2}{|c|}{ all year round } & 7 \\
\hline 4 & Endive & 1 & Asteraceae & \multicolumn{2}{|c|}{ all year round } & 9 \\
\hline 5 & Collards & 2 & Brassicaceae & February & September & 32 \\
\hline 6 & Broccoli & 2 & Brassicaceae & February & October & 20 \\
\hline 7 & Cauliflower & 2 & Brassicaceae & March & October & 18 \\
\hline 8 & Beet & 3 & Chenopodiaceae & February & September & 11 \\
\hline 9 & Spinach & 3 & Chenopodiaceae & February & September & 20 \\
\hline 10 & Zucchini & 4 & Cucurbitaceae & October & February & 14 \\
\hline 11 & Pumpkin & 4 & Cucurbitaceae & November & January & 19 \\
\hline 12 & Cucumber & 4 & Cucurbitaceae & September & March & 13 \\
\hline 13 & Garlic & 5 & Alliaceae & March & April & 24 \\
\hline 14 & Onion & 5 & Alliaceae & March & July & 24 \\
\hline 15 & Leek & 5 & Alliaceae & April & April & 12 \\
\hline 16 & Okra & 6 & Malvaceae & November & January & 27 \\
\hline 17 & Tomato & 7 & Solanaceae & \multicolumn{2}{|c|}{ all year round } & 24 \\
\hline 18 & Carrot & 8 & Apiaceae & \multicolumn{2}{|c|}{ all year round } & 16 \\
\hline 19 & Parsley & 8 & Apiaceae & October & February & 21 \\
\hline 20 & Black velvet bean & 9 & Leguminosae & October & January & 16 \\
\hline 21 & Jack bean & 9 & Leguminosae & October & February & 12 \\
\hline 22 & Lupine & 9 & Leguminosae & March & July & 18 \\
\hline 23 & Hairy vetch & 9 & Leguminosae & March & July & 20 \\
\hline
\end{tabular}

Table 6: Data for the 23 crops: name, botanic family, appropriate planting periods and production time 


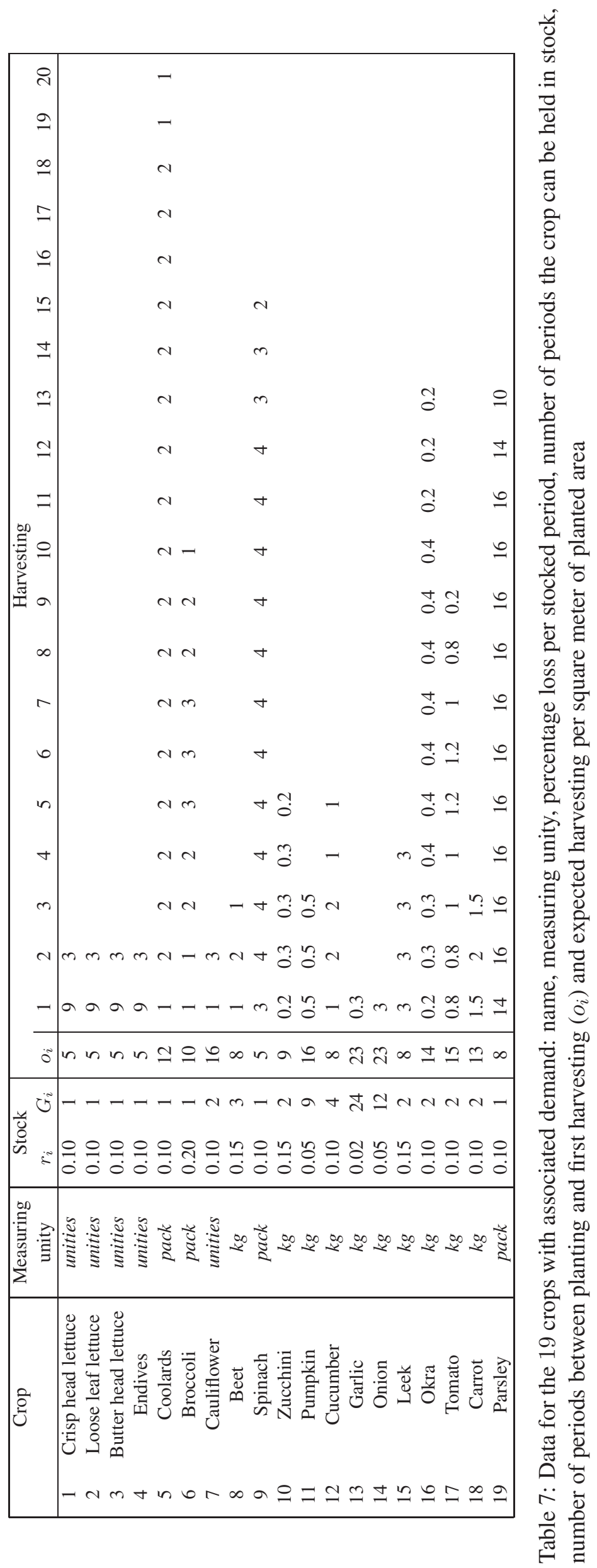




\section{References}

Ahumada, O. and Villalobos, J. R. (2009). Application of planning models in the agri-food supply chain: A review, European Journal of Operational Research 196: 1-20.

Alem, D. J., Munari, P. A., Ferreira, P. A. V. and Arenales, M. N. (2010). On the cutting stock problem under stochastic demand, Annals of Operations Research 179: 169-186.

Alfandari, L., Lemalade, J. L., Nagih, A. and Plateau, G. (2010). A MIP flow model for crop-rotation planning in a context of forest sustainable development, Annals of Operations Research (Forthcoming, doi: 10.1007/s10479-009-0553-0A.) .

Altieri, M. A. (1995). Agroecology: the science of sustainable agriculture, Boulder: Westview press.

Avriel, M. and Williams, A. C. (1970). The value of information and stochastic programming, Operations Research 18: 947-954.

Bachinger, J. and Zander, P. (2006). ROTOR, a tool for generating and evaluating crop rotations for organic farming systems., European Journal Agronomy 26: 130-143.

Bertsimas, D. and Sim, M. (2004). The price of robustness, Operations Research 52: 35-53.

Birge, J. R. (1995). Models and model value in stochastic programming, Annals of Operations Research 59: 1-18. Models for planning under uncertainty.

Birge, J. R. and Louveaux, F. (1997). Introduction to stochastic programming, Springer-Verlag, New York.

Costa, A. M., Cordeau, J.-F. and Laporte, G. (2009). Models and branch-and-cut algorithms for the Steiner tree problem with revenues, budget and hop constraints, Networks 53: 141-159.

Dantzig, G. (1955). Linear programming under uncertainty, Management Science 1: 197-206.

Detlefsen, N. and Jensen, A. L. (2007). Modelling optimal crop sequences using network flows, Agricultural Systems 94: 566-572.

Du Merle, O., Villeneuve, D., Desrosiers, J. and Hansen, P. (1999). Stabilized column generation, Discrete Mathematics 194: 229-237.

El-Nazer, T. and McCarl, B. A. (1986). The choice of crop rotation: A modeling approach and case study, American Journal of Agricultural Economics 68: 127-136.

Escudero, L. F., Garín, A., Merino, M. and Pérez, G. (2007). The value of the stochastic solution in multistage problems, TOP 15: 48-64.

Gliessman, S. R. (2000). Agroecology: ecologycal processes in sustainable agriculture, Chelsea: Ann Arbor.

Gouveia, L. (1999). Using hop-indexed models for constrained spanning and Steiner tree models, in B. Sansò and P. Soriano (eds), Telecommunications Network Planning, Kluwer, Boston, Kluwer, Boston, pp. 21-32. 
Haneveld, W. K. and Stegeman, A. W. (2005). Crop succession requirements in agricultural production planning, European Journal of Operations Research 166: 406-429.

Hildreth, C. G. and Reiter, S. (1951). On the choice of a crop rotation plan, in T. Koopmans (ed.), Proceedings of the Conference on Linear Programming held in Chicago in 1949, pp. 177-188.

ILOG (2006). ILOG CPLEX 10.1 Reference Manual., ILOG.

Jones, J., Hoogenboom, G., Porter, C., Boote, K., Batchelor, W., Hunt, L., Wilkens, P., Singh, U., Gijsman, A. and Ritchie., J. (2003). The DSSAT cropping system model, European Journal of Agronomy 18: 235-265.

Kall, P. and Wallace, S. (1994). Stochastic Programming, Wiley, Chichester etc.

Kantorovich, L. (1960). Mathematical methods of organizing and planning production (translated from a report in Russian, dated 1939), Management Science 6: 366-422.

Liua, C., Fana, Y. and Ordònez, F. (2009). A two-stage stochastic programming model for transportation network protection, Computers \& Operations Research 36: 1582-1590.

Lübbecke, M. E. and Desrosiers, J. (2005). Selected topics in column generation, Operations Research 53: $1007-1023$.

Makatouni, A. (2002). What motivates consumers to buy organic food in the UK? results from a qualitative study, British Food Journal 104: 345-352.

Mulvey, J., Vanderbei, R. and Zenios, S. (1995). Robust optimization of large-scale systems, Operations Research 43: 264-281.

Ong'wen, O. and Wright, S. (2007). Small farmers and the future of sustainable agriculture, Ecofair trade Dialogue, Discussion Paper n.7.

Santos, L. M. R., Costa, A. M., Arenales, M. N. and Santos, R. H. S. (2010). Sustainable vegetable crop supply problem, European Journal of Operational Research 204: 639-647.

Santos, L. M. R., Michelon, P., Arenales, M. N. and Santos, R. H. S. (2010). Crop rotation scheduling with adjacency constraints, Annals of Operations Research (Forthcoming, doi: 10.1007/s10479008-0478-z.) .

Stöckle, C. O., Donatelli, M. and Nelson, R. (2003). CropSyst, a cropping systems simulation model, European Journal of Operations Research 18: 289-307. 


\section{University Library}

\section{- M M N E R VA A gateway to Melbourne's research publications}

Minerva Access is the Institutional Repository of The University of Melbourne

Author/s:

Costa, AM;dos Santos, LMR;Alem, DJ;Santos, RHS

Title:

Sustainable vegetable crop supply problem with perishable stocks

Date:

2014-08-01

Citation:

Costa, A. M., dos Santos, L. M. R., Alem, D. J. \& Santos, R. H. S. (2014). Sustainable vegetable crop supply problem with perishable stocks. ANNALS OF OPERATIONS RESEARCH, 219 (1), pp.265-283. https://doi.org/10.1007/s10479-010-0830-y.

Persistent Link:

http://hdl.handle.net/11343/282667 